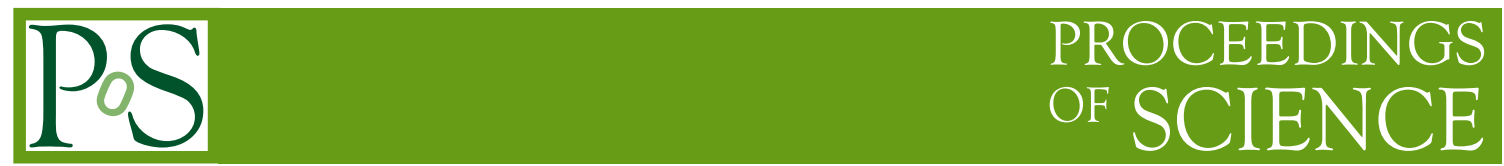

\title{
Hot QCD and warm dark matter
}

\section{Laine*}

Faculty of Physics, University of Bielefeld, D-33501 Bielefeld, Germany

E-mail: laine@physik.uni-bielefeld.de

One of the possible explanations for the dark matter needed in the standard cosmological model is so-called warm dark matter, in the form of right-handed ("sterile") neutrinos with a mass in the $\mathrm{keV}$ range. I describe how various properties of $\mathrm{QCD}$ at temperatures of a few hundred $\mathrm{MeV}$ play an important role in the theoretical computations that are needed for consolidating or falsifying this scenario. In particular the points where lattice QCD could help are underlined.

XXIVth International Symposium on Lattice Field Theory

July 23-28, 2006

Tucson, Arizona, USA

${ }^{*}$ Speaker. 


\section{Introduction}

With the on-going heavy ion collision program at RHIC and the forth-coming one at LHC, a significant amount of resources is being devoted to the study of hadronic matter at very high energy densities. Yet, it is sometimes difficult to express the ultimate physics goals that these programs attempt to meet in a conceptually clean manner. After all, QCD is well-established as a theory of strong interactions - the Lagrangian is known - and the computational challenges that remain for its practical solution will surely ultimately be overcome through lattice simulations and improved analytic approximation schemes, without the need to bother about the background effects and non-equilibrium features that may hamper the setup in heavy ion collision experiments.

Of course, it might be that the ultimate motivation for the heavy ion program does not lie within QCD phenomenology but elsewhere. In documents directed towards the general public, in particular, it is often argued that the main aim is to recreate for a moment the conditions that existed in the very Early Universe. In other words, one is hoping to produce a state which is to a good approximation thermalised, with a temperature in the range of a few hundred $\mathrm{MeV}$, whereby the results should be of interest to the cosmology community.

It is a fact, though, that most cosmologists have shown relatively little interest in the physics related to the QCD epoch since the early 1990's. The main reason is that in order to create cosmological relics, i.e. something that could be astronomically observed today (say, baryonic matter, dark matter, inhomogeneities in the various matter distributions), one needs to deviate from thermodynamical equilibrium. This does not happen easily in cosmology, though, given that the expansion rate is very small compared with the rates of microscopic processes. In fact, for strongly interacting particles, the only possibility of a deviation from equilibrium is through the existence of a first order phase transition. But as lattice studies have been indicating since a long time already (for a recent study, see ref. [1]), the transition in physical QCD seems to be an analytic crossover rather than a real phase transition (or, at most, an extremely weak first order transition). Such a scenario does not appear to lead to any direct cosmological relics (for a recent review, see ref. [2]).

It turns out, however, that this argumentation may be overly pessimistic. In some sense the situation is analogous to the case that will be met in the $p p$-experiments at the LHC: the goal is to find "New Physics", be it a Higgs or something more exotic, but QCD still plays an instrumental role, because it produces a significant background that needs to be understood extremely well.

As a first example in this direction, let us mention the properties of the primordial gravitational wave background. It is assumed that inflation generates a background with a certain "flat" spectrum. The spectrum observed today depends, however, also on the history of the Universe after the inflationary period; in particular it depends on how long a certain wavelength spends inside the horizon after re-entering it. This in turn is dictated by the thermal history and the equation-ofstate of the matter filling the Universe. Consequently, the QCD epoch, during which the expansion rate changes by a significant amount, does lead to a distinctive feature in the gravitational wave spectrum that could be observed today [3] (if our instruments were precise enough).

Another example, closer to the topic of this talk, is the problem of Dark Matter (DM). Again, the main signatures are the determination of the DM relic density and, ultimately, the discovery of DM particles. As we will recall in the next section, however, the thermodynamics of the QCD epoch does play an important role in the computations determining the relic density. 


\section{The problem of Dark Matter}

A number of independent observations, ranging from the length scales of galaxies (rotation curves), through those of galaxy clusters (lensing, large-scale structure), up to cosmological scales (anisotropies in the microwave background), have been consistently making a case for the existence of non-baryonic DM for quite a while already. The amount of DM is estimated to be about $20 \%$ of the total energy density, with a relative error of at most 5\% [4]. The error gets constantly reduced through new observations, and is expected to reach the $1 \%$ level through the Planck mission at the latest. Of course, all of these observations are indirect, and involve some uncontrolled systematic errors. Still, the facts that very different types of observations produce consistent numbers, and that the outcome has remained stable for a long time already, make a reasonably credible case for the existence of DM.

It may be amusing to recall the DM relic density in absolute units as well. At the current moment in the Universe expansion, it amounts to

$$
e_{\mathrm{DM}} \approx 1.1 \frac{\mathrm{GeV}}{\mathrm{m}^{3}}
$$

This may appear to be a small number, but is in fact large compared with the current average density of the total of all known forms of matter, namely

$$
e_{\text {Baryon }} \approx 0.2 \frac{\mathrm{GeV}}{\mathrm{m}^{3}} .
$$

Given that all existing evidence for DM is based on its gravitational interactions, the more precise nature of DM remains unknown. The most popular candidate is so-called Cold Dark Matter $(\mathrm{CDM})$, consisting of particles with a mass $m \gtrsim 10 \mathrm{GeV}$, related perhaps to supersymmetry or supergravity. These particles can be called Weakly Interacting Massive Particles, WIMPs. The hope would then be to discover CDM not only through astronomical observation, but also through directly creating WIMPs in future collider experiments like the LHC.

Unfortunately, as of today, there is no concrete evidence in favour of CDM. Therefore the field remains open for other candidates as well. In this talk I will concentrate on right-handed "sterile" neutrinos as candidates for DM. Within the see-saw scenario it is commonplace to introduce at least two families of massive right-handed neutrinos in any case, to explain the experimental fact that neutrinos have a mass. It is then natural to assume that there are in fact three families of righthanded neutrinos, like there are of the other particles, and that the lightest among these, with a mass in the $\mathrm{keV}$ range, could possible act as DM [5, 6]. Because of the small mass, and subsequently large average energy at any given temperature, such dark matter is referred to as Warm Dark Matter (WDM) in contrast to CDM.

Nevertheless, it must be kept in mind the Nature might also have chosen an even more exotic explanation for what we conceive as DM than just some novel particles: maybe the problems are on the side of gravity, and it is a modification of the theory of gravitational interactions which explains the features that we associate with DM.

Now, let us return to particle dark matter, and recall briefly why hot QCD does play a role in the determination of its relic density, even though the DM particles themselves are very weakly interacting (otherwise, they would not be "dark"). For CDM, for instance, the relic density is 
determined through the moment when the WIMPs decouple, i.e., when their interaction rate becomes smaller than the rate of Universe expansion. The expansion rate, in turn, does depend on the properties of all the particles in the plasma, and most of them do feel strong interactions. It turns out, in particular, that WIMPs of mass $m$ decouple at $T_{\mathrm{dec}} \sim m / 25$ (see, e.g., ref. [7]). For $m=$ $10 \ldots 1000 \mathrm{GeV}, T_{\mathrm{dec}}=0.4 \ldots 40 \mathrm{GeV}$, which indeed is a range where quarks and gluons dominate the equation-of-state (EOS). Therefore the QCD EOS does affect the CDM relic density on a level which is on par with the observational accuracy of the future experiments [8,9].

For right-handed WDM neutrinos, the production mechanism is somewhat different from that for CDM: these particles do not simply decouple, but their production rate really peaks at a certain temperature. The temperature in question depends on the mass of the WDM neutrino, but for the $\mathrm{keV}$ range mentioned above (see Fig. 4 for the observational constraints which make this range to be the relevant one), the peak temperature is around $T \sim 200 \mathrm{MeV}$ [6] (see also Sec. 5). This is just the temperature scale of the QCD phase transition or crossover, so that indirect QCD effects on the WDM relic density are even more dramatic than on the CDM one. It is these effects that will concern us in the following.

\section{Minimal model for right-handed neutrinos}

It has perhaps already become apparent that the philosophies behind the CDM and WDM scenarios are rather different. In the CDM case, the hope is that WIMPs exist and offer a window to genuinely new physics - the more exotic, the better! In the case of right-handed neutrinos, in contrast, the philosophy is to be as down-to-earth as possible: the Minimal Standard Model (MSM) is completed by adding only those degrees of freedom which are necessary in any case for explaining the established experimental facts concerning neutrino oscillations. Otherwise the guiding principle, the construction of the most general renormalizable Lagrangian invariant under the gauge symmetry $\mathrm{SU}(3)_{C} \times \mathrm{SU}(2)_{L} \times \mathrm{U}(1)_{Y}$, remains the same as in the MSM.

Given these principles, the new Lagrangian reads

$$
\mathscr{L}=\mathscr{L}_{\mathrm{MSM}}+\frac{1}{2} \tilde{\bar{N}}_{s}\left[i \not \partial-M_{s}\right] \tilde{N}_{s}-\left[h_{\alpha s} \bar{L}_{\alpha} \tilde{\phi} a_{R} \tilde{N}_{s}+\text { H.c. }\right],
$$

where the generation indices $s, \alpha=1,2,3$ are summed over; $\tilde{N}_{s}$ are Majorana fields; $M_{s}$ are real Majorana masses; $h_{\alpha s}$ are complex Yukawa couplings; $L_{\alpha}$ are lepton doublets; and $\tilde{\phi} \equiv i \tau_{2} \phi^{*}$ is the conjugate Higgs doublet. Because of electroweak symmetry breaking, active neutrinos have masses in this model. We will work in the corner of the parameter space where the masses are given by the see-saw formula as usual, $m_{v_{\alpha}} \sim\left|h_{\alpha s}\right|^{2} v^{2} / M_{s}$, where $v \simeq 246 / \sqrt{2} \mathrm{GeV} \approx 174 \mathrm{GeV}$ is the Higgs field vacuum expectation value.

Now, there is nothing particularly new about the Lagrangian in Eq. (3.1): it is the very theory that is practically always taken as the starting point for the description of massive neutrinos. Usually, though, the assumption is made that the Yukawa couplings $h_{\alpha s}$ are of the same order of magnitude as the known ones, $\left|h_{\alpha s}\right| \lesssim 1$. To reproduce neutrino masses in the range suggested by the oscillation experiments, leads then to the assumption that $M_{1}, M_{2}, M_{3} \sim 10^{10} \ldots 10^{15} \mathrm{GeV}$. Thereby the active neutrino masses are viewed as a window to GUT-scale physics.

Esthetically, though, a theory with such parameter values may look a bit strange: the Higgs mass parameter is in any case expected to be of the order of the electroweak scale, so one may ask 
why the Majorana masses should behave any differently. Clearly, it is possible to keep the active neutrino masses fixed, if a decrease in the Majorana masses is accompanied by a decrease of the neutrino Yukawa couplings in the proportion dictated by the see-saw formula. In refs. $[10,11]$, it was indeed proposed to consider Majorana masses in the range $M_{1} \sim \mathrm{keV}, M_{2}, M_{3} \sim \mathrm{GeV}$, and Yukawa couplings in the ranges $\left|h_{\alpha 1}\right| \lesssim 10^{-11},\left|h_{\alpha 2}\right|,\left|h_{\alpha 3}\right| \lesssim 10^{-7}$. Moreover, it was pointed out that this possibility leads to some phenomenological benefits compared with the usual choice, particularly that the right-handed neutrino $N_{1}$ now has a lifetime long enough to serve as a candidate for DM. The right-handed neutrinos $N_{2}, N_{3}$ are the ones that induce the observed active neutrino mass differences through the see-saw formula, and they also participate in baryogenesis; however, they decay too fast to serve as DM (in fact, they are constrained to decay before the Big Bang Nucleosynthesis epoch [12]). Following refs. [10,11], we will refer to the extension of the MSM with such parameter choices as the " $v$ MSM".

Given that the Yukawa couplings are small and that consequently the right-handed neutrinos interact extremely weakly, we will refer to them as "sterile" neutrinos in the following. For future reference, we also define the mixing angles $\theta_{\alpha 1}$ as

$$
\theta_{\alpha 1} \equiv \frac{h_{\alpha 1} v}{M_{1}}
$$

The mixing angles to be considered in the following are all small, $\left|\theta_{\alpha 1}\right| \lesssim 10^{-4}$.

\section{Primordial production of right-handed neutrinos}

Even though sterile neutrinos interact very weakly, they do get produced in reactions taking place in the Early Universe. This leads to a non-zero primordial abundance, as well as a number of potential consequences:

1. The sterile neutrinos are not stable but decay, for instance through the channel $N \rightarrow v \gamma$. If their life-time is long enough, this results in an X-ray signal which could be observable today.

2. The sterile neutrinos are massive and thus carry a certain energy density. Provided, again, that their lifetime is long enough, this could lead to a contribution to the WDM that plays a role in structure formation.

3. Finally, the various $\mathrm{CP}$-violating scatterings of the sterile neutrinos might contribute towards the baryon asymmetry that exists today.

It is worth stressing that the conventional case of heavy Majorana masses, $M_{1}, M_{2}, M_{3} \sim$ $10^{10} \ldots 10^{15} \mathrm{GeV}$, also leads to a certain primordial abundance and, as is well-known, to the possibility of generating a baryon asymmetry through the mechanism of thermal leptogenesis [13]. This region of the parameter space does not lead to the first two consequences, however, since the heavy neutrinos decay too fast to be still around today, as sources of X-rays and DM.

Because of these potential consequences, it is important to determine the abundance of the sterile neutrinos as a function of their mass $M_{1}$ and mixing angle $\theta_{\alpha 1}$, and we now turn to this computation. Afterwards, the result of this theoretical computation can be confronted with the 
observational constraints that follow from the absence of any visible X-ray signal and from known properties of large-scale structure (cf. Sec. 8).

The basic mechanism for the creation of WDM neutrinos is through inelastic scatterings of light particles existing in the thermal plasma. These processes were first analysed in detail in ref. [6], and a number of refinements can be found in refs. [14]-[17]. Our analysis is based on ref. [18], where the equations entering the production rate were derived directly from quantum field theory, allowing for a systematic investigation of all the effects that play a role, to all orders in the strong coupling constant.

It turns out that, as already mentioned and as demonstrated later on explicitly, the production rate peaks at temperatures of the order of $T \sim 200 \mathrm{MeV} \ll m_{W}$. Therefore, the basic physics that plays a role can be understood within the Fermi model. We start by sketching the ideas with a few graphs, then discuss the general form of the equations that appear; however, the precise details are left out and can be consulted in ref. [18].

An example of a scattering leading to the production of a sterile neutrino $N_{1}$ is shown by the diagram

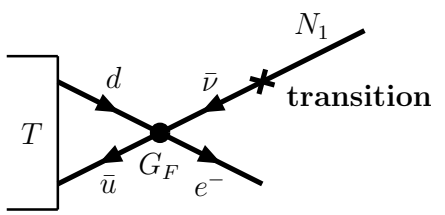

After their production $N_{1}$ are essentially inert: their average lifetime is longer that the age of the Universe, and their density is much below the equilibrium value. Therefore, the $N_{1}$ produced essentially escape the thermal system, just like photons or dilepton pairs produced in heavy ion collision experiments do.

Now, the rate for the production is proportional to the absolute value squared of the amplitude. For the process above, this corresponds to the imaginary part of the 2-point function of active neutrinos:

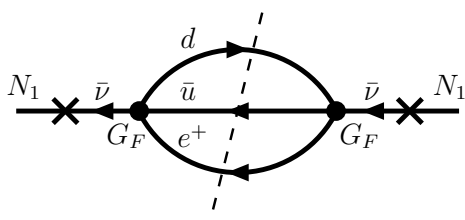

where the dashed line indicates that the cut, or imaginary part, is to be taken.

At temperatures of the order of $200 \mathrm{MeV}$, however, quarks interact strongly, and a perturbative evaluation of the 2-loop diagram in Eq. (4.2) is hardly a good approximation. Fortunately, it is possible to express its contents in a more general way, whereby the quark lines can be combined to a propagator corresponding to flavour singlet or non-singlet vector or axial current correlators [18]:

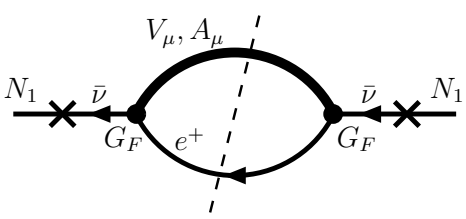

The vector and axial current parts of this graph can be evaluated also beyond QCD perturbation theory, for instance by using chiral perturbation theory or lattice techniques (although the latter are faced with the usual problems related to analytic continuation). 
Finally, there would obviously also be a simpler 1-loop graph that is in principle relevant, namely

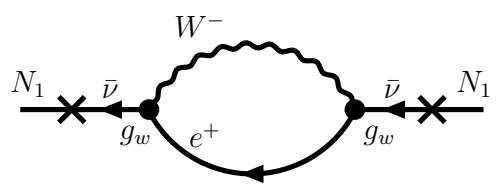

The imaginary part of this graph is, however, exponentially suppressed by $\sim \exp \left(-m_{W} / T\right)$, and thus insignificant at the temperatures that we are interested in. On the other hand this graph does produce a real part (for the real part intermediate particles do not need to be on-shell so that no $\exp \left(-m_{W} / T\right)$ appears), which indeed plays an important role, as we will see.

Let us now express these graphs as formulae [18]. Denoting by $n_{1}$ the number density of the lightest sterile neutrinos $N_{1}$, by $s(T)$ the total entropy density, by $e(T)$ the total energy density, and by $c_{s}^{2}(T)$ the speed of sound squared, the production equation can be written as

$$
-T \frac{\mathrm{d}}{\mathrm{d} T}\left[\frac{n_{1}(T)}{s(T)}\right]=\frac{1}{3 c_{s}^{2}(T) s(T)} \sqrt{\frac{3 m_{\mathrm{Pl}}^{2}}{8 \pi e(T)}} \int \mathrm{d}^{3} \mathbf{q} R(T, \mathbf{q}),
$$

where $m_{\mathrm{Pl}}$ is the Planck mass. The rate, $R(T, \mathbf{q})$, contains in turn the mixing angles and the properties of the self-energy $\Sigma_{\alpha}$ of active neutrinos of flavour $\alpha$ :

$$
R(T, \mathbf{q}) \sim \frac{n_{\mathrm{F}}\left(q^{0}\right)}{(2 \pi)^{3} 2|\mathbf{q}|} \sum_{\alpha=e, \mu, \tau} \theta_{\alpha 1}^{2} \frac{M_{1}^{4} \operatorname{Tr}[\emptyset \operatorname{Im} \not{\not} \alpha]}{\left[M_{1}^{2}+2|\mathbf{q}| \operatorname{Re} \Sigma_{\alpha}\right]^{2}} .
$$

Here we made use of the fact that sterile neutrinos are on-shell, $\left(q^{0}\right)^{2}-\mathbf{q}^{2}=M_{1}^{2}$. Eqs. (4.5), (4.6) show that we need to determine $\operatorname{Re} \Sigma_{\alpha}, \operatorname{Im} \Sigma_{\alpha}$, as well as the thermodynamic quantities $e(T), s(T)$, and $c_{s}^{2}(T)$, in order to estimate $n_{1}(T)$.

Let us start by considering the real and imaginary parts of the active neutrino self-energy. The real part originates from the 1-loop graph shown in Eq. (4.4); a straightforward computation followed by an expansion in $1 / m_{W}^{2}$ leads to the result $[19,20]$

$$
\begin{aligned}
& \operatorname{Re} \Sigma_{\alpha}=Q a_{\alpha}(Q)+u b_{\alpha}(Q), \quad u=(1, \mathbf{0}) \\
& b_{\alpha}(Q)=\frac{16 G_{F}^{2} T^{4}}{\pi \alpha_{w}} q^{0}\left[2 \phi\left(\frac{m_{l_{\alpha}}}{T}\right)+\cos ^{2} \theta_{\mathrm{w}} \phi\left(\frac{m_{v_{\alpha}}}{T}\right)\right],
\end{aligned}
$$

where $\phi$ is a simple dimensionless function, which can easily be evaluated numerically. The function $a_{\alpha}(Q)$ can in fact be ignored, since this part of the self-energy is subdominant compared with the tree-level term $Q$.

Two interesting remarks can be made related to Eq. (4.8). First of all the parametrically leading term, $\sim 1 / m_{W}^{2}$, vanishes. Therefore the result has a prefactor $G_{F}^{2} \sim g_{w}^{4} / m_{W}^{4}$ (of course only two weak gauge couplings $g_{w}$ appear, whereby we have to divide by $\alpha_{w}$ after this normalization). Second, it turns out that if there are non-zero leptonic chemical potentials in the system, $\mu_{L} \neq 0$, then the leading term, $\sim 1 / m_{W}^{2}$, no longer vanishes, but produces a term $b_{\alpha}(Q) \sim-G_{F} n_{L}$, where $n_{L}$ is the lepton density [19]. This leads to the possibility of a "pole", or resonance, in Eq. (4.6), whereby the production rate can be enhanced by a significant amount [21]. Under normal circumstances, however, it is to be expected that the lepton density is of the same order of magnitude as the baryon density, $n_{L} \sim 10^{-10} T^{3}$, in which case these effects are insignificant and can be ignored. 
As far as the imaginary part of the active neutrino self-energy is concerned, its general structure is

$$
\operatorname{Im} \not_{\alpha}(Q) \sim G_{F}^{2} \int \frac{\mathrm{d}^{3} \mathbf{r}}{(2 \pi)^{3}} \mathscr{K}\left(\frac{|\mathbf{q}|}{T}, \frac{|\mathbf{q}+\mathbf{r}|}{T}\right) \gamma^{\mu}(\not+\not R) \gamma^{v} \rho_{\mu \nu}^{V, A}(|\mathbf{q}+\mathbf{r}|-|\mathbf{q}|, \mathbf{r}) .
$$

Here $\mathbf{r}$ is the spatial momentum that flows through the vector or axial current propagator, and $\mathscr{K}$ is a known thermal "kernel", consisting of hyperbolic functions (cf. ref. [18]). The functions $\rho_{\mu \nu}^{V, A}$ represent flavour-singlet and non-singlet vector and axial current spectral functions (i.e., imaginary parts of the retarded two-point functions, $\rho=\operatorname{Im} \Pi_{R}$ ).

Now, the spectral functions contain both leptonic and hadronic contributions. In principle these can be computed perturbatively; this leads to structures familiar from the source terms of Boltzmann equations; for instance, for the case depicted in Eq. (4.2),

$$
\begin{aligned}
& \operatorname{Im} \sum_{\alpha}^{\mathrm{had}}(Q)=4 N_{\mathrm{c}} G_{F}^{2}\left|V_{u d}\right|^{2} n_{\mathrm{F}}^{-1}\left(q^{0}\right) \int \frac{\mathrm{d}^{3} \mathbf{p}_{1}}{(2 \pi)^{3} 2 E_{1}} \int \frac{\mathrm{d}^{3} \mathbf{p}_{2}}{(2 \pi)^{3} 2 E_{2}} \int \frac{\mathrm{d}^{3} \mathbf{p}_{3}}{(2 \pi)^{3} 2 E_{3}} \times \\
& \times\left\{(2 \pi)^{4} \delta^{(4)}\left(P_{1}+P_{2}+P_{3}-Q\right) n_{\mathrm{F} 1} n_{\mathrm{F} 2} n_{\mathrm{F} 3} \mathcal{A}\left(-m_{e}, m_{d},-m_{u}\right)+\right. \\
& +(2 \pi)^{4} \delta^{(4)}\left(P_{2}+P_{3}-P_{1}-Q\right) n_{\mathrm{F} 2} n_{\mathrm{F} 3}\left(1-n_{\mathrm{F} 1}\right) \mathcal{A}\left(m_{e}, m_{d},-m_{u}\right)+Q \\
& +(2 \pi)^{4} \delta^{(4)}\left(P_{1}+P_{3}-P_{2}-Q\right) n_{\mathrm{F} 1} n_{\mathrm{F} 3}\left(1-n_{\mathrm{F} 2}\right) \mathcal{A}\left(-m_{e},-m_{d},-m_{u}\right) \\
& +(2 \pi)^{4} \delta^{(4)}\left(P_{1}+P_{2}-P_{3}-Q\right) n_{\mathrm{F} 1} n_{\mathrm{F} 2}\left(1-n_{\mathrm{F} 3}\right) \mathcal{A}\left(-m_{e}, m_{d}, m_{u}\right)+ \\
& +(2 \pi)^{4} \delta^{(4)}\left(P_{1}-P_{2}-P_{3}-Q\right) n_{\mathrm{F} 1}\left(1-n_{\mathrm{F} 2}\right)\left(1-n_{\mathrm{F} 3}\right) \mathcal{A}\left(-m_{e},-m_{d}, m_{u}\right)+ \\
& +(2 \pi)^{4} \delta^{(4)}\left(P_{2}-P_{1}-P_{3}-Q\right) n_{\mathrm{F} 2}\left(1-n_{\mathrm{F} 1}\right)\left(1-n_{\mathrm{F} 3}\right) \mathcal{A}\left(m_{e}, m_{d}, m_{u}\right)+ \\
& +(2 \pi)^{4} \delta^{(4)}\left(P_{3}-P_{1}-P_{2}-Q\right) n_{\mathrm{F} 3}\left(1-n_{\mathrm{F} 1}\right)\left(1-n_{\mathrm{F} 2}\right) \mathcal{A}\left(m_{e},-m_{d},-m_{u}\right)+ \\
& \left.+(2 \pi)^{4} \delta^{(4)}\left(-P_{1}-P_{2}-P_{3}-Q\right)\left(1-n_{\mathrm{F} 1}\right)\left(1-n_{\mathrm{F} 2}\right)\left(1-n_{\mathrm{F} 3}\right) \mathcal{A}\left(m_{e},-m_{d}, m_{u}\right)\right\}, \mathbb{2}_{2}^{1}
\end{aligned}
$$

where

$$
\mathcal{A}\left(m_{e}, m_{d}, m_{u}\right) \equiv \gamma^{\mu}\left(\not P_{1}+m_{e}\right) \gamma^{\nu} \operatorname{Tr}\left[\left(\not P_{2}+m_{d}\right) \gamma_{\mu} a_{L}\left(\not P_{3}+m_{u}\right) \gamma_{\nu} a_{L}\right] .
$$

The hadronic contributions should, however, really be evaluated beyond perturbation theory in the temperature range of interest, as we have already stressed.

\section{Why does the production rate peak at $T \sim 200 \mathrm{MeV}$ ?}

Let us recall at this point why the production rate of Eq. (4.6) peaks at temperatures of the order of a few hundred $\mathrm{MeV}$ [6].

We have already seen that the real part of the active neutrino self-energy is of the order $\operatorname{Re} \Sigma_{\alpha} \sim$ $G_{F}^{2} T^{4} \alpha_{w}^{-1}|\mathbf{q}|$ (note that $q^{0} \sim|\mathbf{q}|$ for $|\mathbf{q}| \gg M_{1}$ as will be the case here). The imaginary part is more complicated, but ignoring all masses compared with the temperature, it has dimensionally the form $\operatorname{Tr}\left[\not \operatorname{Im} \mathbb{\&}_{\alpha}\right] \sim n_{\mathrm{F}}^{-1}\left(q^{0}\right) G_{F}^{2} T^{6} f(|\mathbf{q}| / T)$, where $f$ is a non-trivial dimensionless function, numerically of order unity. It turns out that the rate peaks at momenta of order $|\mathbf{q}| \sim T$, whereby we can replace $f$ by a number of order unity. Thereby the rate becomes

$$
R(T, \mathbf{q}) \propto \sum_{\alpha=e, \mu, \tau} \theta_{\alpha 1}^{2} \frac{M_{1}^{4} G_{F}^{2} T^{6}}{\left(M_{1}^{2}+100 G_{F}^{2} T^{6}\right)^{2}} .
$$


It is immediately seen that the result is strongly peaked around temperatures where the two terms in the denominator are of similar orders of magnitude, i.e.

$$
T \sim\left(\frac{M_{1}}{10 G_{F}}\right)^{\frac{1}{3}} \sim 200 \mathrm{MeV}\left(\frac{M_{1}}{1 \mathrm{keV}}\right)^{\frac{1}{3}} .
$$

Thus, for sterile neutrino masses in the $\mathrm{keV}$ range, the production rate accidentally coincides with more or less the QCD scale.

\section{The role of the QCD equation-of-state}

Having analysed $\operatorname{Re} \Sigma_{\alpha}$ and $\operatorname{Im} \Sigma_{\alpha}$, we still need to discuss the thermodynamic functions $c_{s}^{2}(T)$, $s(T)$ and $e(T)$, in order to be able to integrate Eq. (4.5).

We parameterise the energy density $e(T)$ and the entropy density $s(T)$ through effective numbers of bosonic degrees of freedom,

$$
e(T) \equiv g_{\mathrm{eff}}(T) \frac{\pi^{2} T^{4}}{30}, \quad s(T) \equiv h_{\mathrm{eff}}(T) \frac{2 \pi^{2} T^{3}}{45} .
$$

The energy and entropy densities follow both from the thermodynamic pressure $p(T)$ through standard relations, $e(T)=T p^{\prime}(T)-p(T)$ and $s(T)=p^{\prime}(T)$. Furthermore the speed of sound squared can be written as

$$
c_{s}^{2}(T) \equiv \frac{p^{\prime}(T)}{e^{\prime}(T)}=\frac{p^{\prime}(T)}{T p^{\prime \prime}(T)} .
$$

In the non-interacting limit, $c_{s}^{2}(T)$ equals $1 / 3$. To summarise, we need reliable estimates of $p(T)$, $p^{\prime}(T)$ and $p^{\prime \prime}(T)$ in order to determine the sterile neutrino abundance from Eq. (4.5).

A reliable determination of $p(T)$ is a long-standing challenge for finite-temperature field theory. Again, leptonic contributions can be well treated in perturbation theory, while hadronic contributions, which dominate the structure in $p(T)$ in the temperature range of interest, are in general hard to compute precisely.

Certain limiting values of $p(T)$ are understood better, though. At low temperatures, $T \lesssim 100 \mathrm{MeV}$, confinement and chiral symmetry breaking guarantee that the system is composed of weakly interacting massive hadrons. Treating them as a "gas" of resonances, one can at very low temperatures approximate

$$
p(T) \approx \sum_{i} T^{4}\left(\frac{m_{i}}{2 \pi T}\right)^{\frac{3}{2}} e^{-\frac{m_{i}}{T}}
$$

and at somewhat higher temperatures replace this with the corresponding relativistic formulae for bosons and fermions, respectively. Even though this prescription is rather phenomenological, lattice simulations suggest that it may work surprisingly well even up to $T \sim 200 \mathrm{MeV}$ [22].

For high temperatures on the other hand, $T \gtrsim 1000 \mathrm{MeV}$, asymptotic freedom guarantees that the system can be viewed as a collection of weakly interacting quarks and gluons:

$$
p(T) \approx \frac{\pi^{2} T^{4}}{90}\left[2\left(N_{\mathrm{c}}^{2}-1\right)+\frac{7}{2} N_{\mathrm{c}} N_{\mathrm{f}}\right]\left(1+\ldots+O\left(g^{6}\right)\right)
$$



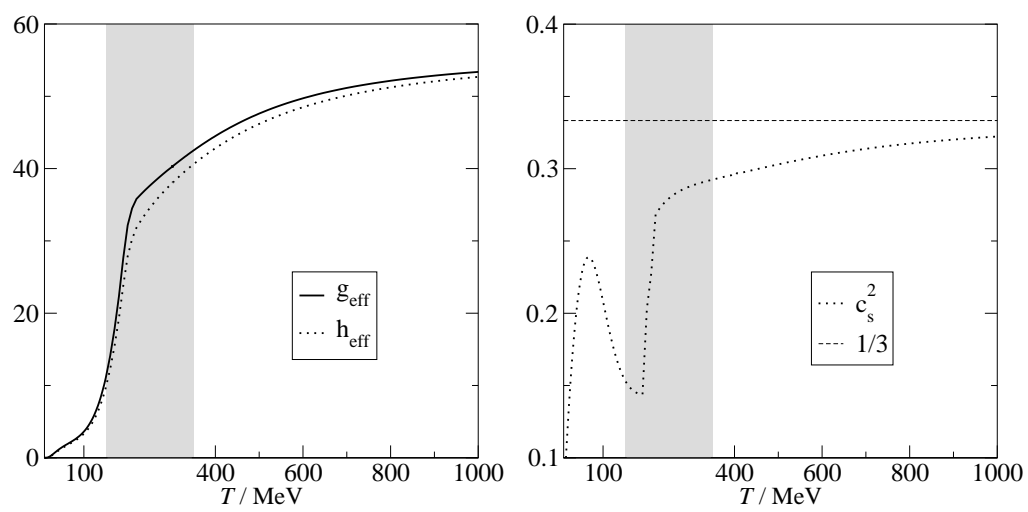

Figure 1: Left: $g_{\text {eff }}, h_{\text {eff }}$ as defined in Eq. (6.1), for $N_{\mathrm{f}}=4$ QCD with physical quark masses [25]. Right: the speed of sound squared $c_{s}^{2}$, for the same system. The shaded region is the range of temperatures where our recipe is purely phenomenological and needs to be improved through future lattice simulations.

where $N_{\mathrm{c}}=3$ is the number of colours and $N_{\mathrm{f}}$ is the number of massless flavours that play a role at the temperatures we are interested in. Perturbative corrections are known up to 4-loop order, $O\left(g^{6}\right)[23,24]$, apart from a single missing coefficient. Moreover, quark mass effects, important for phenomenological applications, have also been studied [25].

In between these two limits the situation is much more complicated. Ideally, one would like to make use of lattice simulations of the type in Refs. [26]-[29]. Unfortunately, it appears that the present results are reliable in a fairly narrow temperature range only; for instance, the characteristic peak that can be seen in $c_{s}^{2}$ at around $T \sim 70 \mathrm{MeV}$, due to light pions, is nowhere to be seen in the existing simulations. In fact there is even no sign of its right slope at temperatures down from the critical one, but the simulations display rather a much deeper dip (down to $\sim 0.1$ ) around the critical region, and then rise at most slightly as the temperature is lowered.

For these reasons, the results that will be presented in the following adhere to the procedure introduced in ref. [25], rather than to lattice simulations. This procedure makes use of a gas of hadronic resonances at low temperatures; the most advanced (up to resummed 4-loop level [23]) weak-coupling results at high temperatures; and an interpolation thereof at intermediate temperatures. ${ }^{1}$ Remarkably, the temperature interval where an interpolating function is needed in order to sew together the two asymptotic functions is fairly narrow, certainly not more than $50 \mathrm{MeV}$, and centered around $T \approx 200 \mathrm{MeV}$, in curiously good agreement with the crossover temperature $T_{\mathrm{c}} \simeq 192 \pm 8 \mathrm{MeV}$ as suggested by recent large scale lattice simulations [30].

The results that follow from this recipe for the quantities that are important for us are illustrated in Fig. 1 for the QCD part, and in Fig. 2 for the whole MSM. In the latter case we have displayed, for completeness, the results in a very broad temperature range.

\section{Numerical results}

With the ingredients discussed, Eq. (4.5) can be solved numerically, as a function of the temperature. From the result, $n_{1}(T) / s(T)$, we can derive the current energy density, $M_{1} n_{1}(T)$, relative

\footnotetext{
${ }^{1}$ We have corrected a minor error in the numerical results of ref. [25].
} 

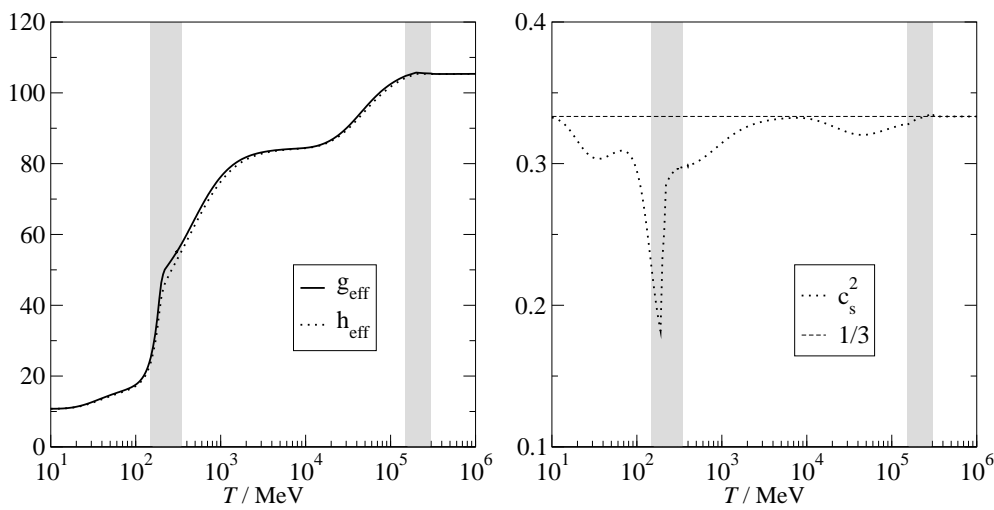

Figure 2: Left: $g_{\text {eff }}, h_{\mathrm{eff}}$ as defined in Eq. (6.1), for $m_{H}=150 \mathrm{GeV}$ [25]. Right: the speed of sound squared $c_{s}^{2}$, for the same system. The shaded regions are the ranges of temperatures where our recipe is purely phenomenological and needs to be improved through future lattice simulations.

to the current entropy density. Finally, the current entropy density, whose value is well-known [4], can be traded for the current critical energy density. Thereby we obtain the parameter $\Omega_{N_{1}}$, characterising the fraction of the current energy density that is carried by sterile neutrinos. This result can then be compared with the observed value for $\Omega_{\mathrm{DM}}$.

In Fig. 3 we show an example of a solution, for specific parameter values [31]. We have here considered the contribution from the active flavour $\alpha=e$ only. It can be observed that the sterile neutrinos indeed get generated at temperatures of a few hundred $\mathrm{MeV}$, and that their relic density can be of an order of magnitude which is relevant for the explanation of DM.

On the other hand, as can be seen in Fig. 3, the current results contain relatively significant hadronic uncertainties. These originate primarily from two sources. First of all, as discussed in Section 4, the imaginary part of the active neutrino self-energy contains the spectral functions of vector and axial currents, which involve poorly understood hadronic effects. It is of course clear that at low enough temperatures there are no hadronic scatterings taking place, since all hadrons are massive. At the same time, at high enough temperatures, the hadronic contributions can be computed perturbatively. A very conservative way to estimate the "error" that these limiting values leave between them, is simply to take half their difference. In the future this error can hopefully be reduced with the help of lattice simulations; the current status on estimating the spectral functions from the lattice is reviewed in ref. [32].

Second, as discussed in Section 6, the hadronic equation-of-state contains significant uncertainties in the temperature range of interest. It turns out that the most important effect as far as the current computation is concerned is the location of the (pseudo)critical temperature $T_{\mathrm{c}}$ : whether the production rate peaks above or below this temperature has a large effect on the final result, given that the kinetic equation of Eq. (4.5) is inversely proportional to $h_{\mathrm{eff}} g_{\mathrm{eff}}^{1 / 2}$. We have estimated these uncertainties by rescaling the temperature units by $20 \%$ in either direction, which certainly is a conservative estimate. The current status of lattice determinations of various thermodynamic quantities in the vicinity of $T_{\mathrm{c}}$ is summarised in ref. [33].

The band indicated in Fig. 3 incorporates both of the error sources discussed, and provides for a conservative estimate of the possible hadronic uncertainties in the results. 


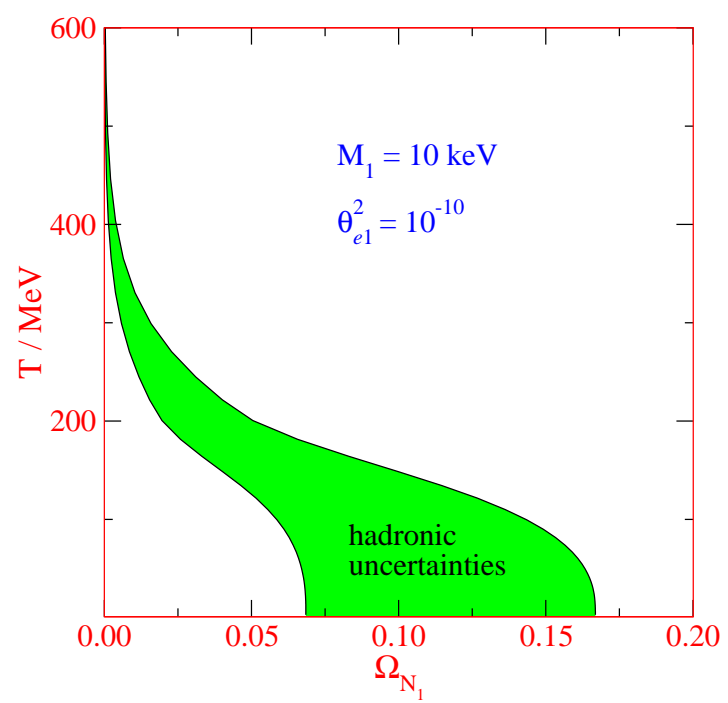

Figure 3: An example of a numerical solution of Eq. (4.5) (according to ref. [31]), normalised with respect to the present total critical energy density $e_{\mathrm{cr}}: \Omega_{N_{1}}(T) \equiv M_{1} n_{1}(T) / s(T) \times s\left(T_{\text {today }}\right) / e_{\mathrm{cr}}\left(T_{\text {today }}\right)$.

\section{Comparison with observational constraints}

We can now confront the theoretical result of Fig. 3 with observational constraints. There are observational constraints from two sides. First of all, if sterile neutrinos with a very small mass constitute all of DM, then structures on small scales tend to be wiped out, compared with structure formation simulations carried out with CDM. Comparing the outcome with actual data, particularly in the form of so-called Lyman- $\alpha$ forest observations, which are sensitive to the smallest distance scales, puts thus a lower bound on the mass of the sterile neutrinos [34]-[37]. It appears that the lower bound could be as high as $14 \mathrm{keV}$ [36].

Second, the heavier the sterile neutrinos are, and the bigger their mixing angles $\left|\theta_{\alpha 1}\right|$ with active neutrinos, the more likely are they to decay. This leads to a characteristic X-ray signal, which however has not been observed. Therefore, it is possible to set an upper bound on a combination of the mass and the mixing angles of the sterile neutrinos [38]-[40].

These two sets of observational constraints are shown in Fig. 4. One can then superimpose curves corresponding to the theoretical computation outlined above. As the final analysis is still in progress [31], we simply show a schematic figure, based on results from earlier analyses, as reviewed in ref. [41]. The dashed curves indicate the effect of varying the lepton density $n_{L} / T^{3}$.

The basic feature to be observed in Fig. 4 is that it appears as if the theoretical computation required a larger mixing angle than experimentally allowed, in order to produce the observed amount of DM (for $n_{L} / T^{3} \sim 10^{-10}$ ). It should be mentioned, however, that the results shown do not include the full analysis as described in this talk. Moreover, it may also be noted that thermal scatterings need not be the only mechanism which produced sterile neutrinos; it could be, for instance, that a certain number density was produced during the inflationary period already [42], to which thermal scatterings would then add their contribution. Therefore, the final judgement on whether it is possible to produce enough DM even in the case of the very small mixing angles allowed by the observational constraints, for the realistic case $n_{L} / T^{3} \sim 10^{-10}$, remains open for the moment. 


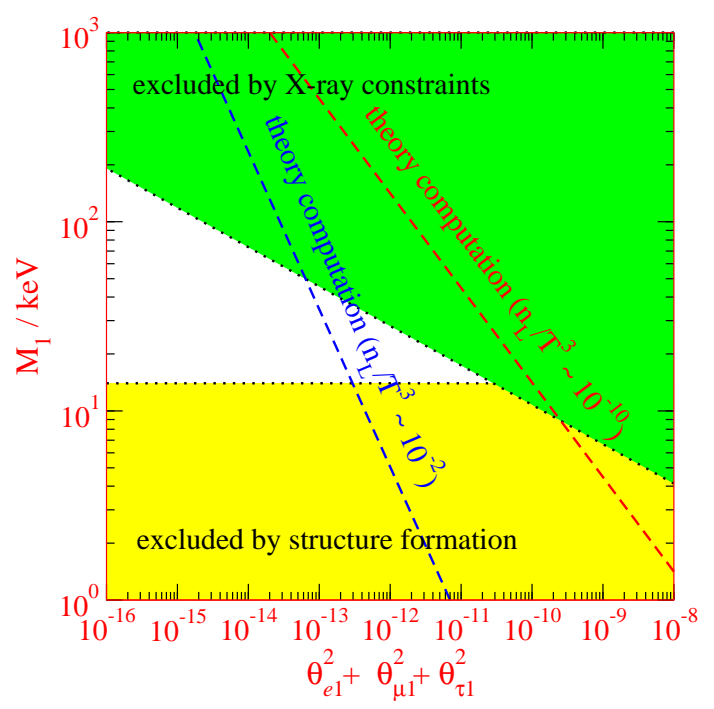

Figure 4: The schematic structure of an exclusion plot comparing observational constraints with theoretical computations. The latter refer to those in the published literature [14]-[17], and do not contain any estimate of the hadronic uncertainties. For a more detailed version of this plot we refer to the recent review in ref. [41].

\section{Conclusions}

The basic scenario of right-handed neutrinos serving as warm dark matter is an old one by now $[5,6]$. It has experienced quite a revival recently, though, because of significant progress both on the observational and on the theoretical sides.

On the observational side, the parameter region (mass, mixing angles) of the lightest righthanded neutrino has been strongly constrained by structure formation and X-ray bounds during the last year or so. This means that attention can now be focussed on a rather specific situation.

On the theoretical side, the scenario of right-handed neutrinos serving as dark matter has taken a more prominent role, thanks to the realization that there is a minimal and natural model, the " $v$ MSM", which not only addresses the dark matter problem, but may also explain neutrino masses [10], baryogenesis [43, 11], and perhaps also various astrophysical problems [44]. Furthermore, the theoretical tools that are needed in the dark matter computation have reached a more mature level [18].

These two sides imply that theoretical computations need to be promoted to a higher level of accuracy than before. As has been underlined in this talk, a fully satisfactory analysis in this respect is only possible once lattice-QCD studies of the QCD equation-of-state and of vector and axial current spectral functions with various flavour structures, produce results with controlled statistical and systematic errors.

\section{Acknowledgments}

This talk is based on refs. [18, 25, 31]; I wish to thank Takehiko Asaka, York Schröder and Misha Shaposhnikov for collaboration. 


\section{References}

[1] P. de Forcrand and O. Philipsen, hep-lat/0607017.

[2] D. Boyanovsky, H.J. de Vega and D.J. Schwarz, hep-ph/0602002.

[3] D.J. Schwarz, Mod. Phys. Lett. A 13, 2771 (1998) [gr-qc/9709027]; N. Seto and J. Yokoyama, J. Phys. Soc. Jap. 72, 3082 (2003) [gr-qc/0305096]; L.A. Boyle and P.J. Steinhardt, astro-ph/0512014.

[4] S. Eidelman et al. [Particle Data Group], Phys. Lett. B 592, 1 (2004).

[5] P.J.E. Peebles, Astrophys. J. 258, 415 (1982);

K.A. Olive and M.S. Turner, Phys. Rev. D 25, 213 (1982).

[6] S. Dodelson and L.M. Widrow, Phys. Rev. Lett. 72, 17 (1994) [hep-ph/9303287].

[7] E.W. Kolb and M.S. Turner, “The Early Universe,” Front. Phys. 69, 1 (1990).

[8] M. Srednicki, R. Watkins and K.A. Olive, Nucl. Phys. B 310, 693 (1988).

[9] M. Hindmarsh and O. Philipsen, Phys. Rev. D 71, 087302 (2005) [hep-ph/0501232].

[10] T. Asaka, S. Blanchet and M. Shaposhnikov, Phys. Lett. B 631, 151 (2005) [hep-ph/0503065].

[11] T. Asaka and M. Shaposhnikov, Phys. Lett. B 620, 17 (2005) [hep-ph/0505013].

[12] A.D. Dolgov, S.H. Hansen, G. Raffelt and D.V. Semikoz, Nucl. Phys. B 580, 331 (2000) [hep-ph/0002223]; Nucl. Phys. B 590, 562 (2000) [hep-ph/0008138].

[13] M. Fukugita and T. Yanagida, Phys. Lett. B 174, 45 (1986).

[14] A.D. Dolgov and S.H. Hansen, Astropart. Phys. 16, 339 (2002) [hep-ph/0009083].

[15] K. Abazajian, G.M. Fuller and M. Patel, Phys. Rev. D 64, 023501 (2001) [astro-ph/0101524].

[16] K.N. Abazajian and G.M. Fuller, Phys. Rev. D 66, 023526 (2002) [astro-ph/0204293].

[17] K. Abazajian, Phys. Rev. D 73, 063506 (2006) [astro-ph/0511630].

[18] T. Asaka, M. Laine and M. Shaposhnikov, JHEP 06, 053 (2006) [hep-ph/0605209].

[19] D. Nötzold and G. Raffelt, Nucl. Phys. B 307, 924 (1988);

K. Enqvist, K. Kainulainen and J. Maalampi, Nucl. Phys. B 349, 754 (1991); J.C. D’Olivo, J.F. Nieves and M. Torres, Phys. Rev. D 46, 1172 (1992).

[20] C. Quimbay and S. Vargas-Castrillón, Nucl. Phys. B 451, 265 (1995) [hep-ph/9504410].

[21] X. Shi and G.M. Fuller, Phys. Rev. Lett. 82, 2832 (1999) [astro-ph/9810076].

[22] F. Karsch, K. Redlich and A. Tawfik, Eur. Phys. J. C 29, 549 (2003) [hep-ph/0303108].

[23] K. Kajantie, M. Laine, K. Rummukainen and Y. Schröder, Phys. Rev. D 67, 105008 (2003) [hep-ph/0211321].

[24] A. Hietanen, K. Kajantie, M. Laine, K. Rummukainen and Y. Schröder, JHEP 01, 013 (2005) [hep-lat/0412008]; F. Di Renzo, M. Laine, V. Miccio, Y. Schröder and C. Torrero, JHEP 07, 026 (2006) [hep-ph/0605042].

[25] M. Laine and Y. Schröder, Phys. Rev. D 73, 085009 (2006) [hep-ph/0603048].

[26] A. Ali Khan et al. [CP-PACS collaboration], Phys. Rev. D 64, 074510 (2001) [hep-lat/0103028]. 
[27] C. Bernard et al., PoS LAT2005, 156 (2005) [hep-lat/0509053].

[28] Y. Aoki, Z. Fodor, S.D. Katz and K.K. Szabo, JHEP 01, 089 (2006) [hep-lat/0510084].

[29] S. Ejiri, F. Karsch, E. Laermann and C. Schmidt, Phys. Rev. D 73, 054506 (2006) [hep-lat/0512040].

[30] M. Cheng et al., hep-lat/0608013.

[31] T. Asaka, M. Laine and M. Shaposhnikov, in preparation.

[32] T. Hatsuda, these proceedings.

[33] U. Heller, these proceedings.

[34] S.H. Hansen, J. Lesgourgues, S. Pastor and J. Silk, Mon. Not. Roy. Astron. Soc. 333, 544 (2002) [astro-ph/0106108].

[35] M. Viel, J. Lesgourgues, M.G. Haehnelt, S. Matarrese and A. Riotto, Phys. Rev. D 71, 063534 (2005) [astro-ph/0501562].

[36] U. Seljak, A. Makarov, P. McDonald and H. Trac, astro-ph/0602430.

[37] M. Viel, J. Lesgourgues, M.G. Haehnelt, S. Matarrese and A. Riotto, Phys. Rev. Lett. 97, 071301 (2006) [astro-ph/0605706].

[38] A. Boyarsky, A. Neronov, O. Ruchayskiy and M. Shaposhnikov, Mon. Not. Roy. Astron. Soc. 370, 213 (2006) [astro-ph/0512509]; JETP Lett. 83, 133 (2006) [hep-ph/0601098]; astro-ph/0603368;

A. Boyarsky, A. Neronov, O. Ruchayskiy, M. Shaposhnikov and I. Tkachev, astro-ph/0603660.

[39] S. Riemer-Sørensen, S.H. Hansen and K. Pedersen, Astrophys. J. 644, L33 (2006) [astro-ph/0603661].

[40] C.R. Watson, J.F. Beacom, H. Yuksel and T.P. Walker, Phys. Rev. D 74, 033009 (2006) [astro-ph/0605424].

[41] K. Abazajian and S.M. Koushiappas, Phys. Rev. D 74, 023527 (2006) [astro-ph/0605271].

[42] M. Shaposhnikov and I. Tkachev, Phys. Lett. B 639, 414 (2006) [hep-ph/0604236].

[43] E.K. Akhmedov, V.A. Rubakov and A.Y. Smirnov, Phys. Rev. Lett. 81, 1359 (1998) [hep-ph/9803255].

[44] A. Kusenko and G. Segrè, Phys. Rev. Lett. 77, 4872 (1996) [hep-ph/9606428];

G.M. Fuller, A. Kusenko, I. Mocioiu and S. Pascoli, Phys. Rev. D 68, 103002 (2003) [astro-ph/0307267];

M. Barkovich, J.C. D'Olivo and R. Montemayor, Phys. Rev. D 70, 043005 (2004) [hep-ph/0402259];

P.L. Biermann and A. Kusenko, Phys. Rev. Lett. 96, 091301 (2006) [astro-ph/0601004];

J. Stasielak, P.L. Biermann and A. Kusenko, astro-ph/0606435;

A. Kusenko, hep-ph/0609081;

F. Munyaneza and P.L. Biermann, astro-ph/0609388. 